\title{
Towards biodegradable chain-growth polymers and polymer particles Re-evaluation of reactivity ratios in copolymerization of vinyl monomers with cyclic ketene acetal using non-linear regression with proper error analysis
}

\section{Supporting Information}

\author{
Jean-Baptiste Lena, Alexander M. Van Herk*
}

Institute of Chemical and Engineering Sciences, 1 Pesek Road, Jurong Island 627833, Singapore

\section{Measurements of the absolute error in the copolymer composition:}

The residual standard deviation (RSD) were calculated with Equations 9 and 10. The signals and the noise were measured manually from the spectra available in the article (both noise and signal were measured with a rule). This gives only a rough estimation of the $S N R$. When the full set of ${ }^{1} \mathrm{H}$ NMR spectra were not available, we considered that the $S N R$ is proportional to the integral of the signal.

System MDPL/MMA ${ }^{1}$ :

Table S1: error on NMR analyses and on the fraction of MDPL in the copolymer MMA/MDPL

\begin{tabular}{|c|c|c|c|c|c|c|c|}
\hline$f_{\mathrm{MDPL}}$ & $F_{\mathrm{MDPL}}$ & $S N R_{\mathrm{MDPL}}$ & $S N R_{\mathrm{MMA}}$ & $R S D_{\mathrm{MDPL}}$ & $R S D_{\mathrm{MMA}}$ & $R S D\left(F_{\mathrm{MDPL}}\right)$ & $\Delta \mathrm{Abs}$ \\
\hline 0.1 & 0.03 & 4 & 42 & $40.4 \%$ & $1.99 \%$ & $42.2 \%$ & 0.01266 \\
\hline 0.2 & 0.06 & 6 & 50 & $24 \%$ & $1.59 \%$ & $25.6 \%$ & 0.01536 \\
\hline 0.4 & 0.14 & 24 & 46 & $4.07 \%$ & $1.77 \%$ & $5.84 \%$ & 0.008716 \\
\hline 0.7 & 0.29 & 32 & 42 & $2.82 \%$ & $1.99 \%$ & $4.81 \%$ & 0.013949 \\
\hline
\end{tabular}

System MDPL/NEtMI ${ }^{2}$ :

Table S2: error on NMR analyses and on the fraction of NEtMI in the copolymer NEtMI/MDPL

\begin{tabular}{|c|c|c|c|c|c|c|c|}
\hline$f_{\text {NEtMI }}$ & $F_{\text {NEtMI }}$ & $S N R_{\text {NEtMI }}$ & $S N R_{\text {MDPL }}$ & $R S D_{\text {NEtMI }}$ & $R S D_{\text {MDPL }}$ & $R S D\left(F_{\text {NEtMI }}\right)$ & $\Delta \mathrm{Abs}$ \\
\hline 0.2 & 0.5 & 19.4 & 28.4 & $5.35 \%$ & $3.28 \%$ & $8.63 \%$ & 0.04315 \\
\hline 0.3 & 0.5 & 19.4 & 28.4 & $5.35 \%$ & $3.28 \%$ & $8.63 \%$ & 0.04315 \\
\hline 0.4 & 0.53 & 20.6 & 26.7 & $4.95 \%$ & $3.55 \%$ & $8.5 \%$ & 0.04506 \\
\hline 0.5 & 0.49 & 19 & 29 & $5.49 \%$ & $3.20 \%$ & $8.79 \%$ & 0.043071 \\
\hline 0.6 & 0.54 & 20.9 & 26.2 & $4.86 \%$ & $3.64 \%$ & $8.5 \%$ & 0.0459 \\
\hline 0.7 & 0.55 & 21.3 & 25.6 & $4.75 \%$ & $3.75 \%$ & $8.5 \%$ & 0.04675 \\
\hline 0.8 & 0.7 & 27.1 & 17.1 & $3.49 \%$ & $6.28 \%$ & $9.77 \%$ & 0.06839 \\
\hline
\end{tabular}


Hill et al. did not provide the values of $f_{\mathrm{NEtMI}}$ and $F_{\mathrm{NEtMI}}$. The values in table $\mathrm{S} 2$ were estimated from the Mayo-Lewis plot.

System MDO/GMA ${ }^{3}$ :

Table S3: error on NMR analyses and on the fraction of MDO in the copolymer GMA/MDO

\begin{tabular}{|c|c|c|c|c|c|c|c|}
\hline$f_{\mathrm{MDO}}$ & $F_{\mathrm{MDO}}$ & $S N R_{\mathrm{MDO}}$ & $S N R_{\mathrm{GMA}}$ & $R S D_{\mathrm{MDO}}$ & $R S D_{\mathrm{GMA}}$ & $R S D\left(\mathrm{~F}_{\mathrm{MDO}}\right)$ & $\Delta \mathrm{Abs}$ \\
\hline 0.9 & 0.61 & 54 & 12 & $1.44 \%$ & $9.89 \%$ & $11.3 \%$ & 0.07 \\
\hline 0.8 & 0.47 & 30 & 10 & $3.06 \%$ & $12.5 \%$ & $18.6 \%$ & 0.09 \\
\hline 0.75 & 0.41 & 20 & 7 & $5.14 \%$ & $19.7 \%$ & $24.8 \%$ & 0.102 \\
\hline 0.70 & 0.31 & 12 & 12 & $9.89 \%$ & $9.89 \%$ & $19.8 \%$ & 0.06 \\
\hline 0.65 & 0.28 & 14 & 16 & $8.12 \%$ & $6.84 \%$ & $15 \%$ & 0.042 \\
\hline 0.60 & 0.24 & 8 & 10 & $16.6 \%$ & $12.5 \%$ & $29.1 \%$ & 0.07 \\
\hline
\end{tabular}

System MDO/VAc ${ }^{4}$ :

Table S4: error on NMR analyses and on the fraction of MDO in the copolymer VAc/MDO

\begin{tabular}{|c|c|c|c|c|c|c|c|}
\hline$f_{\mathrm{MDO}}$ & $F_{\mathrm{MDO}}$ & $S N R_{\mathrm{MDO}}$ & $S N R_{\mathrm{VAc}}$ & $R S D_{\mathrm{MDO}}$ & $R S D_{\mathrm{VAc}}$ & $R S D\left(F_{\mathrm{MDO}}\right)$ & $\Delta \mathrm{Abs}$ \\
\hline 0.01 & 0.005 & 3 & 33 & $58.3 \%$ & $2.71 \%$ & $61.0 \%$ & 0.0305 \\
\hline 0.05 & 0.03 & 6 & 27 & $24 \%$ & $3.50 \%$ & $27.5 \%$ & 0.00825 \\
\hline 0.1 & 0.06 & 9 & 30 & $14.3 \%$ & $3.06 \%$ & $17.3 \%$ & 0.01038 \\
\hline 0.3 & 0.23 & 24 & 18 & $4.07 \%$ & $5.89 \%$ & $9.96 \%$ & 0.022908 \\
\hline 0.5 & 0.42 & 27 & 9 & $3.50 \%$ & $14.3 \%$ & $12.5 \%$ & 0.0525 \\
\hline 0.7 & 0.60 & 51 & 9 & $1.55 \%$ & $14.3 \%$ & $15.8 \%$ & 0.0948 \\
\hline
\end{tabular}

System BMDO/HEMA-TMS ${ }^{5}$

Table S5: error on NMR analyses and on the fraction of BMDO in the copolymer BMDO/HEMA-TMS

\begin{tabular}{|l|l|l|l|l|l|l|l|}
\hline$f_{\mathrm{BMDO}}$ & $F_{\mathrm{BMDO}}$ & $S N R_{\mathrm{BMDO}}$ & $S N R_{\mathrm{HEMA}-\mathrm{TMS}}$ & $R S D_{\mathrm{BMDO}}$ & $R S D_{\mathrm{HEMA}-\mathrm{TMS}}$ & $R S D\left(F_{\mathrm{BMDO}}\right)$ & $\Delta \mathrm{Abs}$ \\
\hline 0.25 & 0.06 & $2.18 *$ & 21 & $87.7 \%$ & $4.8 \%$ & $92.5 \%$ & 0.0555 \\
\hline 0.5 & 0.15 & 5.45 & 19 & $27.1 \%$ & $5.4 \%$ & $32.5 \%$ & 0.04875 \\
\hline 0.6 & 0.20 & 7.27 & 17.9 & $18.8 \%$ & $5.9 \%$ & $24.7 \%$ & 0.0494 \\
\hline 0.7 & 0.26 & 9.45 & 16.6 & $13.4 \%$ & $6.5 \%$ & $19.9 \%$ & 0.05174 \\
\hline 0.8 & 0.33 & 12 & 15 & $9.9 \%$ & $7.4 \%$ & $17.3 \%$ & 0.05709 \\
\hline
\end{tabular}

*A $S N R=3$ usually corresponds to the limit of detection (LOD). When A $\mathrm{SNR}<3$ is observed, it is not possible to confirm the presence of the signal. Only a maximum of $F_{\text {BMDO }}$ can be estimated. 
However, as the SNR is only roughly estimated in this work, the presence of this signal was assumed and Equation 9 was still used to determine the RSD.

System BMDO/MMA ${ }^{6}$ :

Table S6: error on NMR analyses and on the fraction of BMDO in the copolymer BMDO/MMA

\begin{tabular}{|l|l|l|l|l|l|l|l|}
\hline$f_{\mathrm{BMDO}}$ & $F_{\mathrm{BMDO}}$ & $S N R_{\mathrm{BMDO}}$ & $S N R_{\mathrm{MMA}}$ & $R S D_{\mathrm{BMDO}}$ & $R S D_{\mathrm{MMA}}$ & $R S D\left(F_{\mathrm{BMDO}}\right)$ & $\Delta \mathrm{Abs}$ \\
\hline 0.8 & 0.7 & 213.9 & 69.5 & $0.2 \%$ & $1.0 \%$ & $1.2 \%$ & 0.0084 \\
\hline 0.7 & 0.53 & 161.9 & 108.9 & $0.4 \%$ & $0.6 \%$ & $1.0 \%$ & 0.0053 \\
\hline 0.5 & 0.34 & 103.9 & 152.9 & $0.6 \%$ & $0.4 \%$ & $1.0 \%$ & 0.0034 \\
\hline 0.3 & 0.18 & 55 & 190 & $1.4 \%$ & $0.3 \%$ & $1.7 \%$ & 0.00306 \\
\hline
\end{tabular}

\section{Reactivity between the different monomers present in this work:}

The reactivity of each vinyl monomers with other vinyl monomers and with CKA were compared. Results are presents in Table S7. 
Table S7: Reactivity ratios of copolymerization systems containing each monomers included in this study.

\begin{tabular}{|c|c|c|c|c|c|c|c|c|c|c|c|c|c|c|c|c|}
\hline $\begin{array}{c}\text { Monomers } \rightarrow \\
\text { Radicals } \\
\downarrow\end{array}$ & MMA & $\begin{array}{c}\text { MeOEG } \\
\text { MA }\end{array}$ & NEtMI & St & MA & GMA & NVP & VAc & PFS & $\begin{array}{l}\mathrm{DM} \\
\mathrm{AE} \\
\mathrm{MA}\end{array}$ & HEMA & nBA & $\begin{array}{l}\text { NIPA } \\
\text { Am }\end{array}$ & MDO & BMDO & MDPL \\
\hline MMA & 1 & NA & NA & $0.41^{7}$ & $\begin{array}{c}2.15 \pm \\
0.04^{8}\end{array}$ & $\begin{array}{c}0.71 \pm 0.08 \\
9\end{array}$ & $\begin{array}{c}4.04 \pm \\
0.956^{1} \\
0 \\
\end{array}$ & $26^{11}$ & NA & $\begin{array}{c}0.42 \\
\pm 0.0 \\
2^{12} \\
\end{array}$ & $\mathrm{NA}$ & $\begin{array}{l}2.86 \pm \\
0.32^{13}\end{array}$ & $\mathrm{NA}$ & $3.5^{14}$ & $\begin{array}{c}1.950(- \\
0.473, \\
+1.409)^{6 *} \\
\end{array}$ & $\begin{array}{c}3.551(- \\
0.285 \\
+0.401)^{1 *} \\
\end{array}$ \\
\hline MeOEGMA & NA & 1 & NA & NA & NA & NA & NA & NA & NA & NA & $\mathrm{NA}$ & $\mathrm{NA}$ & NA & NA & NA & $6.95^{15}$ \\
\hline NEtMI & NA & NA & 1 & NA & NA & NA & NA & NA & NA & NA & NA & NA & NA & NA & NA & $\begin{array}{c}0.157(- \\
0.111, \\
+0.221)^{2 *}\end{array}$ \\
\hline St & $0.57^{7}$ & NA & NA & 1 & $0.75^{16}$ & $\begin{array}{c}0.54 \pm 0.07 \\
17\end{array}$ & $\begin{array}{l}17.2 \pm \\
1.55^{18} \\
\end{array}$ & $57.8^{19}$ & NA & NA & $\begin{array}{c}0.332 \pm 0.006 \\
20\end{array}$ & $\begin{array}{l}0.79 \pm \\
0.08^{21}\end{array}$ & $\mathrm{NA}$ & $22.6^{22}$ & $8.53^{23}$ & $\mathrm{NA}$ \\
\hline MA & $0.4 \pm 0.12^{8}$ & NA & NA & $0.18^{16}$ & 1 & NA & NA & $6.38^{24}$ & NA & NA & $\begin{array}{c}- \\
0.005 \pm 0.036 \\
25\end{array}$ & NA & NA & $26.53^{26}$ & NA & NA \\
\hline GMA & $0.52 \pm 0.06^{9}$ & NA & NA & $\begin{array}{c}0.16 \pm 0 \\
03^{17}\end{array}$ & NA & 1 & $\begin{array}{c}4.29 \pm \\
0.498^{2} \\
7 \\
\end{array}$ & $\mathrm{NA}$ & NA & NA & $\mathrm{NA}$ & $\begin{array}{l}2.16 \pm \\
0.57^{28}\end{array}$ & $\mathrm{NA}$ & $\begin{array}{c}4.2351(- \\
0.879 \\
+1.940)^{3 *}\end{array}$ & NA & $\mathrm{NA}$ \\
\hline NVP & $\begin{array}{c}0.066 \pm 3.965 \\
10 \\
\end{array}$ & NA & NA & $\begin{array}{c}0.057 \pm 0 \\
.028^{18} \\
\end{array}$ & $\mathrm{NA}$ & $\begin{array}{c}0.003 \pm 0.0 \\
18^{27} \\
\end{array}$ & 1 & NA & NA & NA & $\mathrm{NA}$ & $\mathrm{NA}$ & $\mathrm{NA}$ & $6.31^{29}$ & $\mathrm{NA}$ & $\mathrm{NA}$ \\
\hline VAc & $0.03^{11}$ & NA & $\mathrm{NA}$ & $0.08^{19}$ & $0.03^{24}$ & NA & $\mathrm{NA}$ & 1 & NA & NA & $\mathrm{NA}$ & $\begin{array}{l}3.48 \pm \\
3.39^{30}\end{array}$ & NA & $\begin{array}{c}1.707(- \\
0.236 \\
+0.500)^{4 *}\end{array}$ & NA & NA \\
\hline PFS & NA & NA & NA & NA & NA & NA & $\mathrm{NA}$ & NA & 1 & NA & NA & NA & NA & NA & $9.9^{31}$ & NA \\
\hline DMAEMA & $1.01 \pm 0.54^{12}$ & NA & NA & NA & NA & $\mathrm{NA}$ & $\mathrm{NA}$ & NA & $\mathrm{NA}$ & 1 & NA & NA & $\mathrm{NA}$ & $\mathrm{NA}$ & $6.96^{32}$ & NA \\
\hline HEMA & NA & NA & NA & $\begin{array}{c}0.856 \pm 0 \\
.018^{20}\end{array}$ & $\begin{array}{l}8.87 \pm \\
8.28^{25}\end{array}$ & $\mathrm{NA}$ & $\mathrm{NA}$ & NA & NA & NA & 1 & $\mathrm{NA}$ & NA & $\mathrm{NA}$ & $\begin{array}{c}4.692(- \\
0.240 \\
+0.305)^{5 *}\end{array}$ & NA \\
\hline $\mathrm{nBA}$ & $\begin{array}{c}0.11 \pm 0.018^{1} \\
3\end{array}$ & NA & NA & $\begin{array}{c}0.25 \pm 0 . \\
05^{21}\end{array}$ & $\mathrm{NA}$ & $\begin{array}{c}0.083 \pm 0.0 \\
52^{28}\end{array}$ & $\mathrm{NA}$ & $\begin{array}{c}0.018 \pm 0 \\
.072^{30} \\
\end{array}$ & NA & NA & NA & 1 & NA & $\mathrm{NA}$ & $3.7^{33}$ & NA \\
\hline NIPAAm & $\mathrm{NA}$ & NA & NA & NA & NA & $\mathrm{NA}$ & $\mathrm{NA}$ & $\mathrm{NA}$ & NA & NA & $\mathrm{NA}$ & $\mathrm{NA}$ & 1 & $\mathrm{NA}$ & $7.31^{34}$ & $\mathrm{NA}$ \\
\hline MDO & $0.04^{14}$ & NA & NA & $0.21^{22}$ & $\begin{array}{c}0.023^{2} \\
6\end{array}$ & $\begin{array}{c}0.155(- \\
0.057 \\
+0.132)^{3}\end{array}$ & $\begin{array}{c}0.014^{2} \\
9\end{array}$ & $\begin{array}{c}0.948(- \\
0.359 \\
+0.759) \\
4 *\end{array}$ & NA & NA & $\mathrm{NA}$ & $\mathrm{NA}$ & $\mathrm{NA}$ & 1 & NA & NA \\
\hline BMDO & $\begin{array}{c}0.503(- \\
0.191 \\
+0.601)^{6 *} \\
\end{array}$ & NA & NA & $1.08^{23}$ & NA & NA & $\mathrm{NA}$ & NA & $\begin{array}{c}0.35 \\
31\end{array}$ & $\begin{array}{c}0.14 \\
32\end{array}$ & $\begin{array}{c}0.019(- \\
0.014 \\
+0.016)^{5 *}\end{array}$ & $0.08^{33}$ & $0.11^{34}$ & NA & 1 & NA \\
\hline MDPL & $\begin{array}{c}0.014(- \\
0.073 \\
+0.092)^{1 *}\end{array}$ & $0^{15}$ & $\begin{array}{c}0.021(- \\
0.069 \\
+0.129)^{2 *}\end{array}$ & NA & NA & NA & NA & NA & NA & NA & NA & NA & NA & $\mathrm{NA}$ & NA & 1 \\
\hline
\end{tabular}



*Reevaluated values from this study ; NA: not available in the literature.

St, styrene; MMA, methyl methacrylate; OEGMA, oligo(ethylene glycol) methacrylate; NEtMI, N-ethyl melamide; MA, methyl acrylate; NVP, $N$-vinylpyrolidone; VAc, vinyl acetate; PFS, 2,3,4,4,6pentafluorostyrene; DMAEMA, $N, N$-dimethylaminoethyl methacrylate; HEMA-TMS, trimethylsilyl hydroxyethyl methacrylate; $n \mathrm{BA}, n$-butyl acrylate; NIPAAm, $N$-isopropylacrylamide.

\section{References:}

1. Tran, J.; Guegain, E.; Ibrahim, N.; Harrisson, S.; Nicolas, J., Efficient synthesis of 2-methylene-4phenyl-1,3-dioxolane, a cyclic ketene acetal for controlling the NMP of methyl methacrylate and conferring tunable degradability. Polymer Chemistry 2016, 7 (26), 4427-4435.

2. Hill, M. R.; Guegain, E.; Tran, J.; Figg, C. A.; Turner, A. C.; Nicolas, J.; Sumerlin, B. S., Radical RingOpening Copolymerization of Cyclic Ketene Acetals and Maleimides Affords Homogeneous Incorporation of Degradable Units. Acs Macro Letters 2017, 6 (10), 1071-1077.

3. Undin, J.; Finne-Wistrand, A.; Albertsson, A. C., Copolymerization of 2-Methylene-1,3-dioxepane and Glycidyl Methacrylate, a Well-Defined and Efficient Process for Achieving Functionalized Polyesters for Covalent Binding of Bioactive Molecules. Biomacromolecules 2013, 14 (6), 2095-2102.

4. Undin, J.; Illanes, T.; Finne-Wistrand, A.; Albertsson, A. C., Random introduction of degradable linkages into functional vinyl polymers by radical ring-opening polymerization, tailored for soft tissue engineering. Polymer Chemistry 2012, 3 (5), 1260-1266.

5. Zhang, Y.; Chu, D. F.; Zheng, M. Y.; Kissel, T.; Agarwal, S., Biocompatible and degradable poly(2hydroxyethyl methacrylate) based polymers for biomedical applications. Polymer Chemistry 2012, 3 (10), 2752-2759.

6. Wickel, H.; Agarwal, S.; Greiner, A., Homopolymers and random copolymers of 5,6-benzo-2methylene-1,3-dioxepane and methyl methacrylate: Structural characterization using 1D and 2D NMR. Macromolecules 2003, 36 (7), 2397-2403.

7. Simionescu, C. I.; Simionescu, B. C.; loan, S., Plasma-induced living radical copolymerization. Journal of Macromolecular Science-Chemistry 1985, A22 (5-7), 765-778.

8. Zubov, V. P.; Valuev, L. I.; Kabanov, V. A.; Kargin, V. A., Effects of complexing agents in radical copolymerization. Journal of Polymer Science Part a-1-Polymer Chemistry 1971, 9 (4), 833-\&.

9. Iwakura, Y.; Kurosaki, T.; Ariga, N.; Ito, T., Copolymerization of methyl methacrylate with glycidyl methacrylate and reaction of copolymer with amines. Makromolekulare Chemie 1966, 97 (SEP), 128-\&.

10. Czerwinski, W. K., Solvent effect on free-radical chain polymerization .7. kinetic-analysis of the binary-system methyl methacrylate/ $\mathrm{N}$-vinyl pyrolidone in bulk and in model solvents in term of the reactant-solvent complex model. Macromolecules 1995, 28 (16), 5411-5418.

11. Bevington, J. C.; Johnson, M., Radical polymerizations involving esters of vinyl alcohol .2. Copolymerizations. European Polymer Journal 1968, 4 (6), 669-+.

12. Orbay, M.; Laible, R.; Dulog, L., Preparation of amide and amine groups containing copolymers of methyl-methacrylate and their performance in solid polymer composites. Makromolekulare ChemieMacromolecular Chemistry and Physics 1982, 183 (1), 47-63.

13. Brosse, J. C.; Gauthier, J. M.; Lenain, J. C., Synthesis of polymers with hydroxyl end groups by radical reactions.11. Study of the co-polymerization of methyl-methacrylate with various acrylates and methacrylates - determination of reactivity ratios. Makromolekulare Chemie-Macromolecular Chemistry and Physics 1983, 184 (3), 505-517. 
14. Agarwal, S., Microstructural characterisation and properties evaluation of poly (methyl methacrylate-co-ester)s. Polymer Journal 2007, 39 (2), 163-174.

15. Delplace, V.; Guegain, E.; Harrisson, S.; Gigmes, D.; Guillaneuf, Y.; Nicolas, J., A ring to rule them all: a cyclic ketene acetal comonomer controls the nitroxide-mediated polymerization of methacrylates and confers tunable degradability. Chemical Communications 2015, 51 (64), 12847-12850.

16. Burlant, W. J.; Green, D. H., Some gamma-ray initiated polymerizations. Journal of Polymer Science 1958, 31 (122), 227-230.

17. Iwakura, Y.; Kurosaki, T.; Nakabayashi, N., Reactive fiber .1. Copolymerization and copolymer of acrylonitrle with glycidyl methacrylate and with glycidyl acrylate. Makromolekulare Chemie 1961, 44-6, 570-590.

18. Bork, J. F.; Coleman, L. E., Nitrogen-containing monomers .2. Reactivity ratios of NVinyloxazolidone and N-vinylpyrolidone with vinyl monomers. Journal of Polymer Science 1960, 43 (142), 413-421.

19. Semchikov, Y. D.; Smirnova, L. A.; Knyazeva, T. Y.; Bulgakova, S. A.; Voskoboinik, G. A.;

Sherstyanykh, V. I., General character of the effect of influence of molecular mass on the composition of copolymer during homogeneous radical copolymerization. Vysokomolekulyarnye Soedineniya Seriya A

1984, 26 (4), 704-710.

20. Okano, T.; Aoyagi, J.; Shinohara, I., Wettability and composition of 2-hydroxyethyl methacrylate copolymers. Nippon Kagaku Kaishi 1976, (1), 161-165.

21. Kostanski, L. K.; Hamielec, A. E., Influence of temperature on butyl acrylate styrene copolymerization parameters. Polymer 1992, 33 (17), 3706-3710.

22. Bailey, W. J.; Endo, T.; Gapud, B.; Lin, Y. N.; Ni, Z.; Pan, C. Y.; Shaffer, S. E.; Wu, S. R.; Yamazaki, N.; Yonezawa, K., Synthesis of functionally-terminated oligomers by free-radical ring-opening polymerization. Journal of Macromolecular Science-Chemistry 1984, A21 (8-9), 979-995.

23. Wickel, H.; Agarwal, S., Synthesis and characterization of copolymers of 5,6-benzo-2-methylene1,3-dioxepane and styrene. Macromolecules 2003, 36 (16), 6152-6159.

24. Mayo, F. R.; Walling, C.; Lewis, F. M.; Hulse, W. F., Copolymerization .5. Some copolymerizations of vinyl acetate. Journal of the American Chemical Society 1948, 70 (4), 1523-1525.

25. Varma, I. K.; Patnaik, S., Copolymerization of 2-hydroxiethyl methacrylate with alkyl acrylates. European Polymer Journal 1976, 12 (4), 259-261.

26. Sun, L. F.; Zhou, R. X.; Liu, Z. L., Synthesis and enzymatic degradation of 2-methylene-1,3dioxepane and methyl acrylate copolymers. Journal of Polymer Science Part a-Polymer Chemistry 2003, 41 (18), 2898-2904.

27. Soundararajan, S.; Reddy, B. S. R., Glycidyl methacrylate and N-vinyl-2-pyrolidone copolymers synthesis, characterization, and reactivity ratios. Journal of Applied Polymer Science 1991, 43 (2), 251 258.

28. Dhal, P. K.; Ramakrishna, M. S.; Babu, G. N., Copolymerization of glycidyl methacrylate with alkyl acrylate monomers. Journal of Polymer Science Part a-Polymer Chemistry 1982, 20 (6), 1581-1585.

29. Choi, S.; Lee, K.; Kwon, S.; Kim, H., Preparation of fine particles of poly(N-vinyl-2-pyrrolidone-co2-methylene-1,3-dioxepane) using supercritical antisolvent. Journal of Supercritical Fluids 2006, 37 (3), 287-291.

30. Nikolaev, A. F.; Vishneve.Lp; Gromova, O. A.; Grigorev.Mm; Kleshche.Ms, Peculiarites of copolymerization of vinyl acetate with butyl acrylate. Vysokomolekulyarnye Soedineniya Section A 1969, 11 (11), 2418-\&.

31. Agarwal, S., Radical ring opening and vinyl copolymerization of 2,3,4,5,6-pentafluorostyrene with 5,6-benzo-2-methylene-1,3-dioxepane: Synthesis and structural characterization using 1D and 2D NMR techniques. Journal of Polymer Research 2006, 13 (5), 403-412. 
32. Agarwal, S.; Ren, L. Q.; Kissel, T.; Bege, N., Synthetic Route and Characterization of Main Chain Ester-Containing Hydrolytically Degradable Poly(N,N-dimethylaminoethyl methacrylate)-Based Polycations. Macromolecular Chemistry and Physics 2010, 211 (8), 905-915.

33. Huang, J. Y.; Gil, R.; Matyjaszewski, K., Synthesis and characterization of copolymers of 5,6benzo-2-methylene-1, 3-dioxepane and n-butyl acrylate. Polymer 2005, 46 (25), 11698-11706.

34. Ren, L. Q.; Agarwal, S., Synthesis, characterization, and properties evaluation of poly (Nisopropylacrylamide)-co-ester s. Macromolecular Chemistry and Physics 2007, 208 (3), 245-253. 